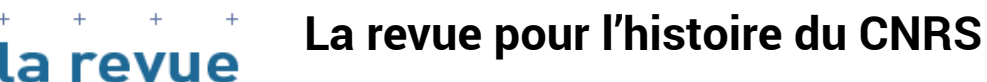

POUR LHISTOIRE DU CNRS $\quad 24 \mid 2009$

Soixante-dixième anniversaire du CNRS

\title{
Histoire de famille
}

Irène Théry

\section{(2) OpenEdition}

Journals

Édition électronique

URL : https://journals.openedition.org/histoire-cnrs/9084

DOI : 10.4000/histoire-cnrs.9084

ISSN : 1955-2408

Éditeur

CNRS Éditions

Édition imprimée

Date de publication : 5 octobre 2009

ISSN : 1298-9800

Référence électronique

Irène Théry, "Histoire de famille », La revue pour l'histoire du CNRS [En ligne], 24 | 2009, mis en ligne le 05 octobre 2009, consulté le 20 mai 2021. URL : http://journals.openedition.org/histoire-cnrs/9084 ; DOI : https://doi.org/10.4000/histoire-cnrs.9084

Ce document a été généré automatiquement le 20 mai 2021.

Comité pour l'histoire du CNRS 


\title{
Histoire de famille
}

\author{
Irène Théry
}

1 L'anthropologie de la parenté a été extrêmement importante dans la constitution même des sciences sociales. Henry Lewis Morgan est considéré comme le fondateur des études sur le sujet. Cet avocat défendait les Indiens contre les expropriations qu'ils subissaient de la part des Blancs au fur et à mesure de la construction du chemin de fer aux États-Unis. Excellent connaisseur de plusieurs tribus, il remarqua la variété des terminologies de parenté, et fit l'hypothèse que celle-ci avait un sens et pouvait faire l'objet d'une étude scientifique. Ses ouvrages ont eu une influence décisive en anthropologie.

2 Mais malheureusement, après la seconde guerre mondiale, on a réorganisé les savoirs en séparant dans le champ académique, la sociologie (assimilée à la science de nos sociétés) et l'anthropologie (science des autres sociétés). Les sociologues ont perdu l'habitude de réfléchir sur la parenté, cependant que les anthropologues travaillaient sur la parenté dans des sociétés lointaines, avec un regard "surplombant" sans véritable réflexivité sur nous-mêmes: "Ce qui me passionne depuis 15 ans, c'est la renaissance des études sur la famille et la parenté liant sociologie, anthropologie et histoire. La grande nouveauté est que les chercheurs enrichissent leur approche de la famille et de ses changements en l'inscrivant à l'intérieur d'un champ de recherche né dans les années 1970, celui des "études de genre ». Or le genre a transformé notre compréhension des systèmes de parenté en montrant qu'ils articulent non seulement alliance, filiation et germanité mais quatre sortes de relations : de même sexe, de sexe opposé, de sexe indifférencié et de sexe combiné », explique- telle.

3 Selon I. Théry, tous les systèmes de parenté connaissent des métamorphoses importantes dans le contexte de la mondialisation. Ainsi, elle collabore régulièrement avec ses collègues anthropologues pour comprendre ces phénomènes contemporains. Parmi eux, on peut citer par exemple Pascale Bonnemère, directrice de recherche au CNRS, qui étudie une société de Papouasie Nouvelle-Guinée, ou Serge Tcherkézoff, directeur d'études à l'EHESS, spécialiste de l'île de Samoa en Polynésie.

4 I. Théry, qui se définit comme une sociologue très imprégnée d'interdisciplinarité, (l'anthropologie, l'histoire, le droit...) sait que son parcours est un peu atypique 
comparé à celui des sociologues de la famille. De formation littéraire, son agrégation de lettres modernes obtenue en 1975, I. Théry enseigne d'abord le français en collège. Son intérêt pour le changement social et en particulier la place sociale des femmes la conduit au métier de chercheuse. Pour elle, la première traduction de l'égalité des sexes est une métamorphose assez radicale de la vie privée et familiale, et des liens de parenté. C'est pourquoi elle a choisi ce thème de recherche alors assez mal vu chez les féministes. Selon I. Théry, la meilleure façon d'observer l'évolution des normes familiales était d'observer la scène judiciaire. Elle est donc devenue sociologue du droit, ce qui lui a permis d'étudier les transformations des normes in vivo. Par là, elle renouait avec Durkheim et les premiers sociologues de l'école française de sociologie, qui connaissaient très bien le droit et les arcanes de la justice. Elle s'est donc penchée sur les archives judiciaires, en a fait sa spécialité et a soutenu sa thèse en 1983 sur "l'usage judiciaire du critère de l'enfant», puis elle est entrée au CNRS dans un laboratoire du ministère de la Justice qui n'existe plus aujourd'hui: le centre de recherches de Vaucresson.

Plus tard, elle fit une grande enquête sur les divorces très conflictuels pour étudier les rapports entre le travail du juge et celui de l'expert psychiatre dans 700 affaires jugées entre 1985 et 1990 tout en préparant un travail collectif sur l'histoire du droit et la Révolution française. Cette double perspective l'amènera à la rédaction de son livre $L e$

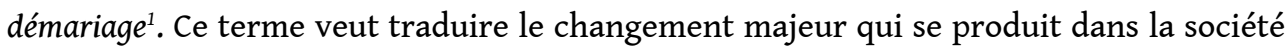
quand le fait de se marier, ne pas se marier ou se démarier, devient une question de conscience personnelle.

6 Le démariage bouleverse la filiation. C'est pour comprendre celle-ci que I. Théry inscrit son travail sur la famille en référence à l'anthropologie de la parenté. Cette perspective lui a permis de montrer qu'un des grands phénomènes liés au démariage était les familles recomposées². Dans la revue Dialogue de 1987-88, elle dirige un numéro spécial «Les beaux enfants » et propose pour la première fois le terme alors inédit de famille recomposée. Depuis, elle a élargi son étude à la compréhension des différentes réformes du droit de la famille, dans une perspective de sociologie législative : « Le droit s'adapte aux changements de nos représentations de la parenté. On a inventé le Pacs et institué en droit le couple de même sexe, ce qui aurait paru « impossible » encore dans les années 1980. Le grand changement depuis ces années-là est la désarticulation entre le couple et la filiation. Ainsi, l'enfant peut-il conserver ses deux parents même s'ils sont séparés. Pour moi, la famille contemporaine, qui est organisée selon des valeurs de liberté, d'égalité, de fraternité, n'est pourtant pas une mini-démocratie. Sa façon de conjuguer le "je » et le "nous » est différente car les relations familiales sont asymétriques, par exemple parents/enfants. Cette famille s'ouvre de plus en plus du côté de l'amitié et c'est nouveau : le droit n'a rien à voir avec la valeur d'amitié ", précise-t-elle.

7 Aujourd'hui, I. Théry essaie d'ajouter une nouvelle corde à son arc en liant la réflexion des sciences humaines et sociales avec celle de la philosophie, en particulier la philosophie contemporaine du langage et de l'action.

Point de vue Irène

Théry pense que les chercheurs ont trop souvent assimilé la famille au moment où sont présents de jeunes enfants. Selon elle, il faut impérativement déplacer l'attention vers la vieillesse. Par exemple, la notion de veuvage a été très peu étudiée et c'est la seule catégorie démographique qui n'a pas subi de modernisation. Un nouvel objet de recherche serait de comprendre l'importance du veuvage hors mariage. I. Théry dirige une thèse sur le sujet : «Nos systèmes statistiques ne tiennent 
pas compte de cette catégorie de la population. Que va faire la société de ces veufs non mariés qui n'ont pas d'existence sociale? Il va falloir sortir cette réalité de l'ombre", souligne-t-elle. En 1998, à la demande du gouvernement, I. Théry a accepté de se glisser dans la peau d'un expert pour établir un rapport sur les principales réformes qui seraient utiles en droit civil de la famille. Elle avait trois mois pour le rendre. Pour elle, ce fut une expérience très enrichissante. Elle n'était pas là pour se substituer au débat de société mais pour apporter des éléments de connaissance et de raisonnement à la réflexion des politiques et des citoyens. I. Théry estime qu'un certain type d'attaques actuelles contre «les experts» sont purement démagogiques. L'expertise a des règles, qui peuvent et doivent être discutées, mais il est important que les chercheurs s'attachent à rendre compte de ce qu'ils font pour nourrir la discussion démocratique et étayer la décision politique. Pour I. Théry, «la tour d'ivoire n'a jamais été une garantie d'objectivité. "

8 Femmes dolganes habillées de vêtements en peau de renne de tradition nganassane. Décor perlé de tradition dolgane. Région de Sopotchnoye, Taïmyr. Région d'Atchaïvaïam, Kamchatka. (C) CNRS Photothèque/Système Renne

\section{NOTES}

1. I. Théry. Le démariage, justice et vie privée. Odile Jacob, 2001.

2. M.-T. Meulders-Klein, I. Théry. Les recompositions familiales aujourd'hui. Nathan, coll. Essais \& recherches, 1993.

\section{RÉSUMÉS}

Pour Irène Théry, directrice d'études à l'École des hautes études en sciences sociales (EHESS), il est important de ne pas couper la sociologie de la famille contemporaine d'une perspective historique et anthropologique. C'était la grande leçon des recherches d'avant-guerre, par exemple celles de l'École française de sociologie dirigée par Durkheim, puis Mauss. L'idée majeure qu'il convient de réaffirmer vigoureusement aujourd'hui est que la particularité des familles humaines par rapport aux familles animales est d'être des groupes toujours institués dans un système de parenté.

\section{AUTEUR}

\section{IRÈNE THÉRY}

Irène Théry est directrice d'études à l'École des hautes études en sciences sociales. 